\title{
Remineralization Potential of a Novel Silver Nanoparticle Rinse on Severely Demineralized Enamel In-vitro: A 14-Day Trial
}

\author{
Callister C, Callister M, Nolan M, Nolan R* \\ Novus Research, Department of Dental Research, USA
}

\begin{abstract}
Although fluoride-based remineralization systems have dominated prevention for the last century, new and innovative approaches are changing the preventative landscape in dental medicine. With the advent of nanotechnology, a wealth of new materials has become available to aid in remineralizing high risk tooth surfaces. While respecting traditional approaches, utilizing silver nanoparticles (AgNPs) for remineralization has unexplored potential for use via novel mechanisms for rehardening enamel surfaces. It was the purpose of this study to examine the remineralization potential of a novel rinse containing silver nanoparticles (AgNPs) on severely demineralized enamel in vitro.
\end{abstract}

Keywords: Hydroxyapatite; Silver nanoparticles; Zero point of charge.

\section{INTRODUCTION}

Nanotechnology has rapidly advanced in the last decade. Although many compounds have been studied, silver nanoparticles (AgNPs) have been a key research focus in dentistry and medicine. This is due to their antimicrobial, antiviral, and antifungal properties [1]. Additionally, multiple studies have shown that AgNPs can not only prevent demineralization, but can also remineralize tooth structure $[2,3]$. It was the purpose of this study to evaluate whether or not a novel rinse containing plant based AgNPs could remineralize severely demineralized enamel. Fluorides mechanism of action is well known and occurs via the replacement of the hydroxyl group in Hydroxyapatite (HAP). This increases HAP crystal density, reducing enamel susceptibility to future acid insults [4]. However, in situations where caries or severe demineralization is already present, remineralization from this mechanism may be limited in nature due to the lack of intact HAP crystal sites. It is the hope that newer systems may offer alternative mechanisms of action which will either be synergistic with fluoride or offer increased remineralization potential. One of the challenges for AgNPs is that their mechanism of action for remineralization in vitro is not well known, and may be size, stability, and coating dependent. Additionally, AgNP action could occur on different portions of the HAP crystal than fluoride. As more research is done, remineralization systems can be further optimized, allowing for better and more sophisticated approaches for prevention of demineralization and caries.

\section{Optimizing prevention strategies}

Although redundancy is often seen with a negative connotation, it is a large part of why saliva is so successful as a preventative medium in the oral cavity. Therefore, it is important that hygiene systems designed to be anti-cariogenic mimic and support the salivary system already in place. Saliva contains multiple layers of redundancy for remineralization, via a reservoir of critical ions and buffering capacity against $\mathrm{pH}$ changes [5]. As a result, oral hygiene systems which have multiple modes of action offer promising opportunities for prevention. This is due to the fact that cariogenic biofilms can be aggressive, unforgiving, and resistant to single modes of action. Therefore, by incorporating multiple modes of action for remineralization, antimicrobial action, and a delivery system for these processes, a result which supports or enhances salivary action could be achieved.

Salivary pH ideally ranges from between 7 to 8 , whereas individuals who lack critical minerals may have a salivary $\mathrm{pH}$ of 6.5 or below [6]. Due to the nature of HAP, attraction of calcium and hydroxide are far superior at a $\mathrm{pH}$ of 7 or above, and decrease exponentially as $\mathrm{pH}$ drops $[7,8]$. Therefore, according to the force of attraction equation for mineral surfaces, and the zero point of charge (ZPC) concept noted by Bell, it stands to reason, that a $\mathrm{pH}$ roughly above 7 is ideal for remineralization [9].

Novel remineralization system

Silver nanoparticles (AgNPs) have long been known for their

"Correspondence to: Ryan Nolan, Chief Scientist Novus Research, Department of Dental Research. 3596 Mountain Vista Parkway, Suite \#3, Provo, UT 84606, USA, Tel: 1-779-206-9804; E-mail: Ryannolandmd@gmail.com

Received: Apr 04, 2020; Accepted: Apr 17, 2020; Published: Apr 24, 2020

Citation: Callister C, Callister M, Nolan M, Nolan R (2020) Remineralization Potential of a Novel Silver Nanoparticle Rinse on Severely Demineralized Enamel In-vitro: A 14-Day Trial. J Nanomed Nanotech. 11:542. doi: 10.35248/2157-7439.20.11.542

Copyright: (C2020 Callister C, et al. This is an open-access article distributed under the terms of the Creative Commons Attribution License, which permits unrestricted use, distribution, and reproduction in any medium, provided the original author and source are credited. 
antimicrobial action, as well as their anti-adherent effects on oral biofilms [10]. AgNPs have also been shown in multiple studies to increase microhardness of HAP and prevent demineralization at high risk sites, such as around orthodontic brackets and composites $[2,3,11]$. However, plant based AgNPs have not been tested for their remineralization capacity in vitro. In addition, due to their small size it has been shown that AgNPs can be used as a delivery system for calcium to enhance uptake into oral biofilms [12]. As a result of their low toxicity, plant based AgNPs are ideal candidates for antimicrobials and remineralization agents in oral care [13]. In addition to AgNPs, xylitol has long been known for its antiacidogenic and anti-adherent properties [14]. At a concentration of $25 \%$ or above, xylitol can act as a salivary stimulant and aid as a carrier system for calcium and phosphate ions, mimicking salivary statherin as a reservoir for critical minerals [14]. Finally, calcium is a well-known component of saliva. It has been known for some time that calcium deficient regions develop at the first stage of demineralization due to the breakdown of the external HAP surfaces [15]. This in turn, weakens HAP, and makes it more susceptible to acid attacks on its phosphate backbone [15]. HAP has three critical ions: calcium, phosphate, and hydroxide. As a result of this, hygiene systems which lack these components and are acidic can in turn lead to dissolution of calcium and phosphate. As $\mathrm{pH}$ increases, more hydroxide is readily present. Therefore, oral hygiene systems with a neutral to alkaline $\mathrm{pH}$ containing calcium, are highly likely to aid in remineralization [16].

\section{Theoretical mechanism of action}

When considering novel remineralization systems which utilize nanoparticles, there are many potential mechanisms of action to consider. With remineralization, the presence of the critical species (calcium, phosphate and hydroxide ions) are absolutely critical. As a result of these ions being present in saliva, small plant based AgNPs present an interesting potential opportunity for crystal formation. Crystal growth occurs in multiple stages, but could begin with nucleation around an AgNP which has anchored to a demineralized site. Due to the AgNP being plant based, it has a polysaccharide (PS) coating, making it negatively charged and able to bind to positively charged demineralized sites. As the AgNP is $<5-10 \mathrm{~nm}>$ it has a large charge to size ratio, allowing it to act as a seed for potential crystal formation. Due to the high stability of these AgNPs in solution, a stable nanoaggregate could form, causing growth in the presence of calcium and phosphate ions. It is suspected that these crystals remain nano in size, and contain an AgNP core. With other known silver containing compounds such as SDF, silver ions react and deposit as silver phosphate, silver hydroxide, and silver chloride [17]. Due to silver ion reaction kinetics, these deposition reactions likely occur too quickly for stable crystal formation to develop, resulting in black staining. This has been shown in multiple studies via elemental analysis and X-Ray Diffraction (XRD) [17,18]. Although elemental analysis or XRD was not done for this study, no staining was noted. This suggests that plant based AgNPs could have an alternative mechanism of action for remineralization through the formation of Ca-P AgNP nanocrystals shown in Figure 1. This mechanism is likely unique to AgNP's with small cores, low burst release and PS coating.

\section{MATERIALS}

Sections of enamel were cut from noncarious $3^{\text {rd }}$ molars and premolars removed from patients during standard orthodontic or preventative care. This study was given IRB exemption as No HIPPA attachments were noted and all teeth were donated anonymously

\section{Disorganized HAP Crystals}
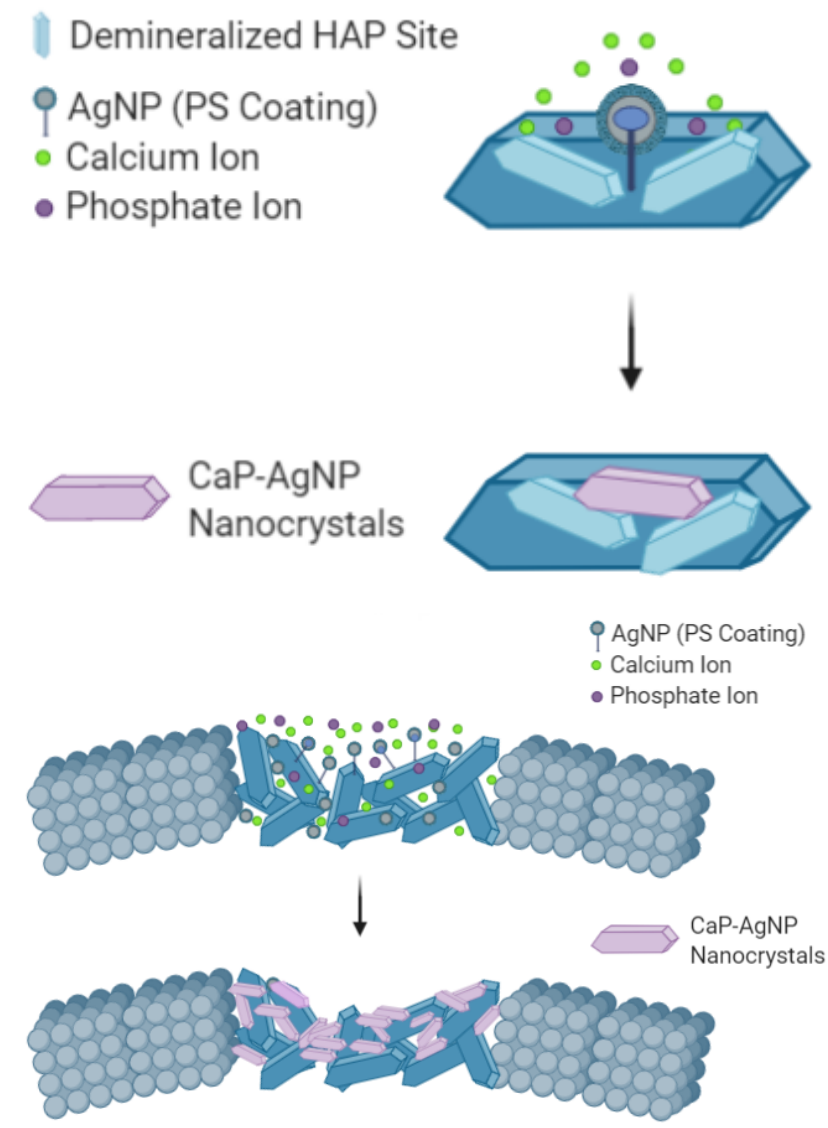

Figure 1: Theoretical mechanism of action for the formation of $\mathrm{CaP}$ -AgNp Nanocrystals.

from local dental offices. All hard and soft tissue deposits from teeth were removed via ultrasonication. Buccal and lingual sections were cut from teeth using a double-sided NTI serrated diamond burr. Samples were cut to be approximately 4 X $6 \mathrm{~mm}$ with 2 $\mathrm{mm}$ thickness. Samples were checked for cracks or abnormalities, sterilized, and placed in $0.1 \%$ thymol solution 48 hours prior to the study. All sections were cut with care, and placed in a $16 \times 16$ $\mathrm{mm}$ circular mould. The back portion of the slabs were bonded using Scotchbond Universal ( $3 \mathrm{M}$ ) and light cured based on the manufacturers recommendation. These samples were then placed into a non-stick disc mould which was filled with nanohybrid composite (HS, Nano Elegance) and cured with a CO2-LED curing light based on the manufacturer's recommendations. All samples were checked and verified to be the same shape and relative size of other samples to ensure uniformity across the groups. The enamel surface was polished which an SL2 Shofu white stone to ensure a flat surface for measurement.

In order to test the novel AgNP rinse, it was necessary to ensure a proper method for control. Artificial saliva was made using USP grade calcium chloride $(1.5 \mathrm{mM})$, potassium phosphate $(0.9 \mathrm{mM})$, potassium chloride $(0.13 \mathrm{mM})$ and potassium hydroxide $(1.78 \mathrm{mM})$ $(\mathrm{pH}=7.0)$. The artificial saliva group was used as a negative control throughout the study to eliminate any potential gain from salivary action alone. The positive control used was a neutral sodium fluoride $(\mathrm{NaF} \quad 0.05 \% \mathrm{w} / \mathrm{v})$ rinse purchased over the counter $(\mathrm{pH}=6.6)$. The test solution was provided by Novus Research and contained xylitol, silver nanoparticles $(0.0015 \% \mathrm{w} / \mathrm{v}$ AgNPs) and calcium acetate $(\mathrm{pH}=7.8)$. The demineralizing solution utilized was USP grade citric acid (1 wt\%), and the storage solution prior to the 
study was thymol $(0.1 \mathrm{wt} \%)$. All test groups remained in artificial saliva throughout the study after demineralization.

\section{METHODS AND TESTING}

TEM \& UV-VIS were taken of the novel AgNP rinse to determine size and distribution of the AgNPs. This is shown in Figures 2 and 3.

In order to test microhardness, an Instron 5969 - dual column tabletop testing system machine was utilized. This was followed by imaging on an SEM Hitachi TM3030 Plus tabletop to clarify the exact indentation measurements. Each sample was indented with $5 \mathrm{~N}$ of force for 1 second. SEM images were taken of each indentation to verify length and width of the indentation. Equation 1-1 was used for microhardness measurements. The distances were measured via SEM in micrometers and converted to $\mathrm{mm}$ for $\mathrm{d} 1$ and $\mathrm{d} 2$.

Equation 1-1: Microhardness calculations Microhardness $=\frac{0.1891 * \text { Force }}{(\mathrm{d} 1) *(\mathrm{~d} 2)}$

\section{EXPERIMENTAL SETUP}

All enamel samples were tested for initial microhardness, followed by demineralization with $1 \mathrm{wt} \%$ citric acid solution for 24 hours in order to severely demineralize the enamel. After this, microhardness was tested again, and all samples were placed in artificial saliva. The negative and positive control and experimental samples were exposed to their respective agents for approximately 10 minutes daily throughout the 14-day experiment after demineralization had occurred. Artificial saliva, was changed regularly to ensure uniformity across samples. Upon removal of samples, they were rinsed in DI water thoroughly. Experimental samples and the positive control were then placed back into artificial saliva throughout the 14-day period. Microhardness was measured at four intervals: initially (predemineralization), post-demineralization, at 7 days and at 14 days
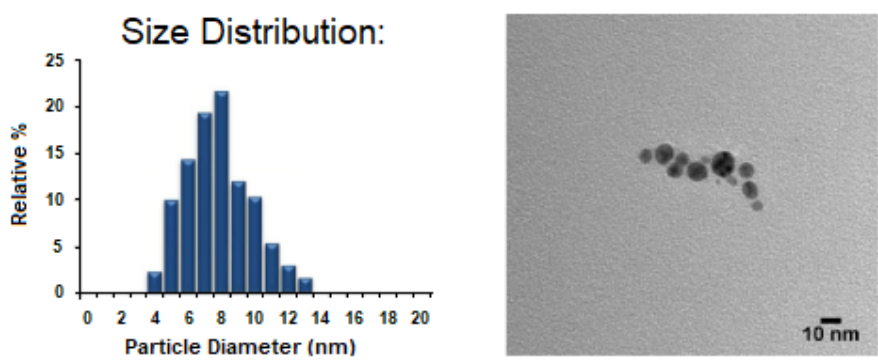

Figure 2: TEM \& Size Analysis of Novel AgNPs.

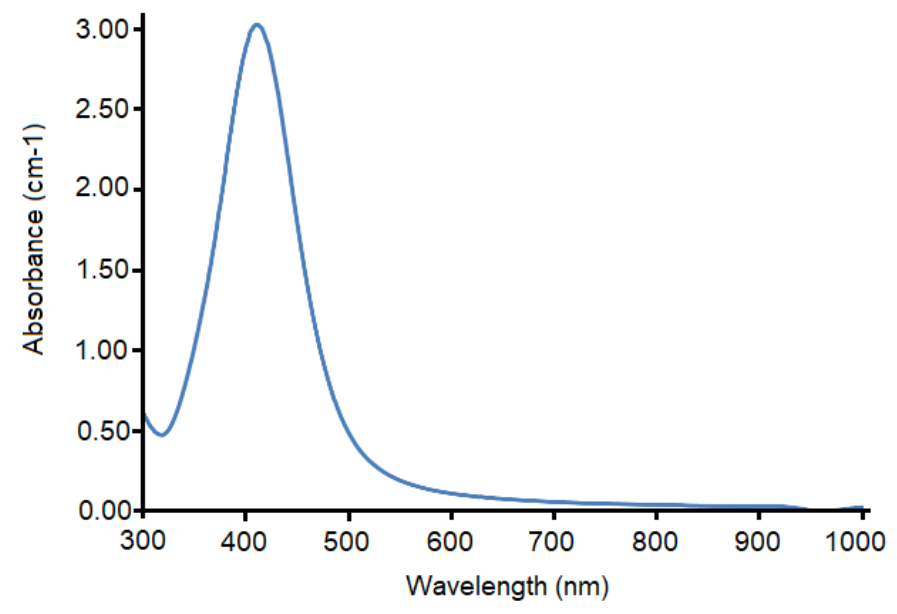

Figure 3: UV-VIS Analysis of AgNP rinse. via an Instron, at $5 \mathrm{~N}$ for $1 \mathrm{~s}$. This was followed by imaging on an SEM. All samples were visually checked via SEM and externally for cracks or marginal breakdown (Figure 4). Samples which had macro cracks or cracks running through the indentations did not meet the criteria and were not used in the study.

\section{RESULTS}

In the case of the severely demineralized samples, an average microhardness below 20 for all groups was achieved utilizing $1 \mathrm{wt} \%$ citric acid. Sample images before and after demineralization show the severity of damage to the enamel and the HAP microlattice. This can be seen in Figures 5 and 6 .

It can be noted that as demineralization occurs, the surface texture of the enamel completely changes, and indentations become much larger, requiring less magnification to visualize. After demineralization, the regular striations and surface morphology of HAP became rough, chalky and disorganized. This is due to the finite amount of structural integrity left in the HAP units in the demineralized enamel. Table 1 shows the microhardness results for each group tested. One-way ANOVA ( $p<0.05$ ) was used for each group to determine significance at 7 days and 14 days compared to the post demineralization values. Significance within the same group is denoted by a star. One-way ANOVA ( $p<0.05$ ) was also used to determine significance between groups at 7 and 14 days based on the net gains from the demineralization stage, denoted by a triangle. It should be noted that no significant difference was noted between the negative control (artificial saliva) and the

\section{Remineralization of Demineralized Enamel:}

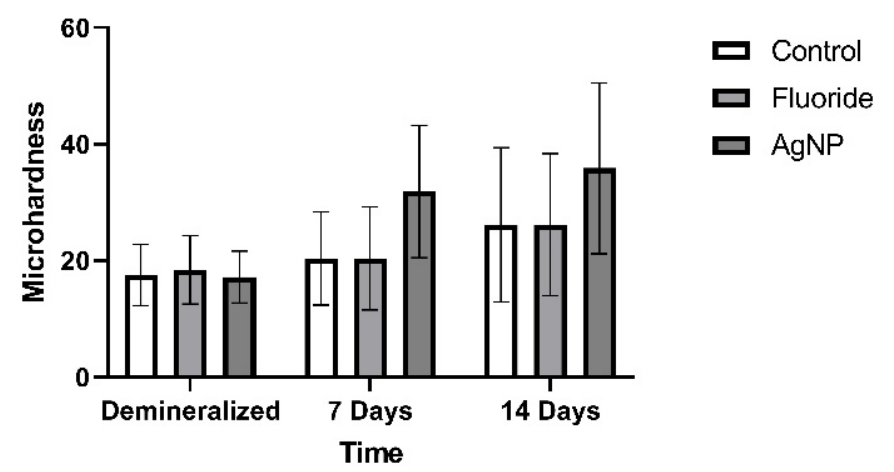

Figure 4: Microhardness changes over time.

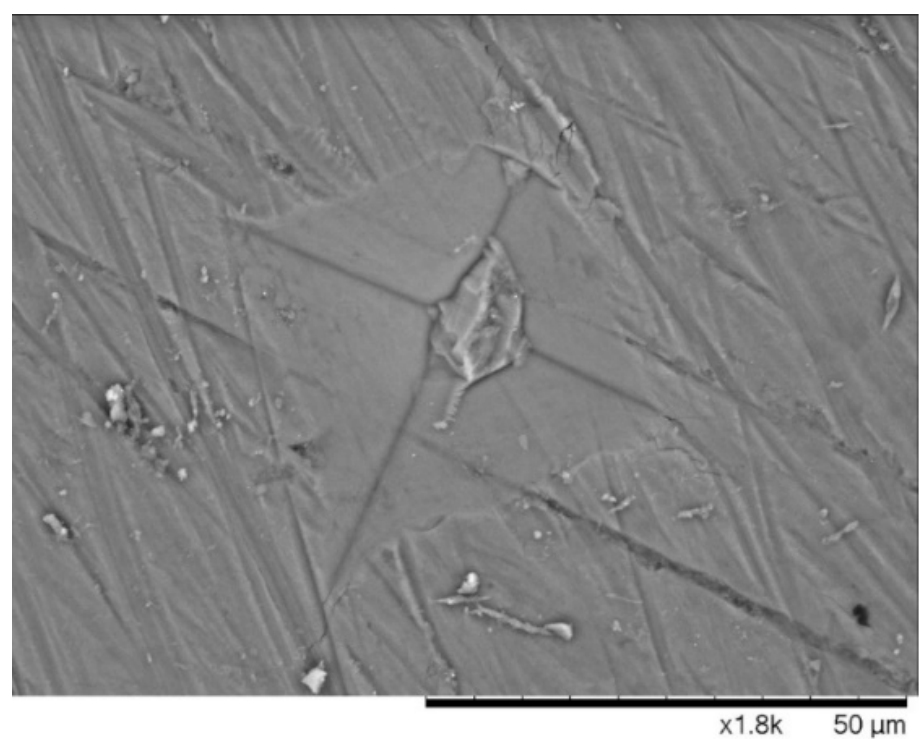

Figure 5: Sample indentation at baseline (pre-demineralization). 


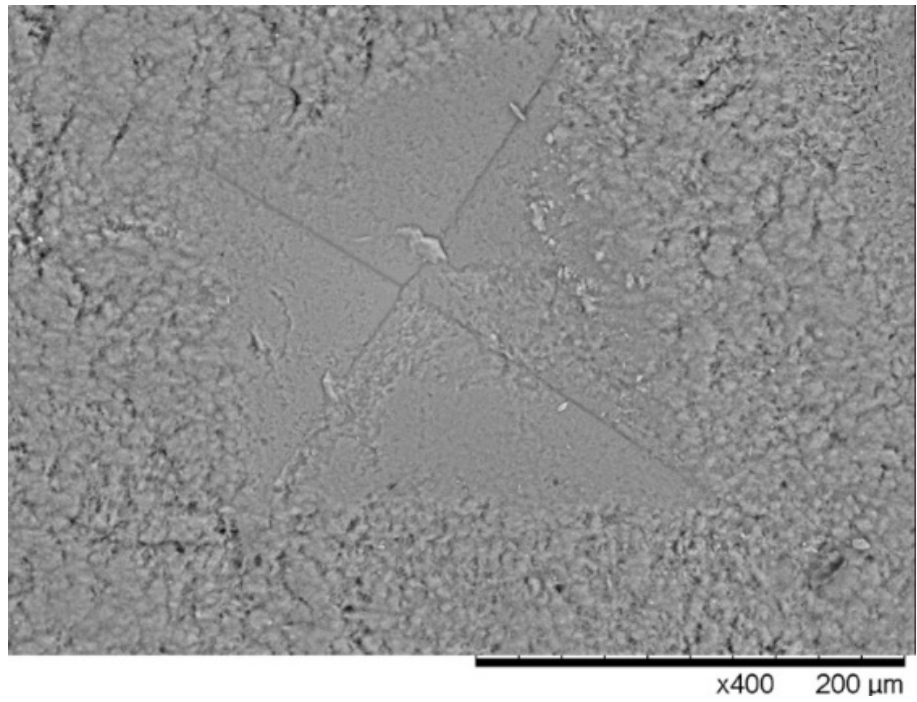

Figure 6: Sample indentation after demineralization (postdemineralization).

Table 1: Microhardness measurements at various time points. *Significant change $\mathrm{p}<0.05$ compared to demineralized values within the same group, $\nabla$ Significant change $\mathrm{p}<0.05$ compared to other groups microhardness changes at the specified time interval.

\begin{tabular}{ccccc}
\hline Groups & Baseline & Demineralized & 7 Days & 14 Days \\
\hline Control & $378.57 \pm$ & $17.52 \pm 5.23$ & $20.33 \pm$ & $26.12^{*} \pm$ \\
$(\mathrm{n}=15)$ & 43.54 & & 8.02 & 13.26 \\
\hline Fluoride rinse & $392.67 \pm$ & $18.38 \pm 5.87$ & $20.34 \pm$ & $26.19^{*} \pm$ \\
$(\mathrm{n}=15)$ & 77.26 & & 8.82 & 12.20 \\
\hline AgNP rinse & $396.61 \pm 71.72$ & $17.16 \pm 4.45$ & $31.85^{*} \nabla \pm$ & $35.85 * \nabla \pm$ \\
$(\mathrm{n}=15)$ & & & 11.37 & 14.66 \\
\hline
\end{tabular}

fluoride group at all time points. However, each individual group saw a significant gain at 14 days compared to their demineralized baselines. The AgNP group saw significance at 7 and 14 days from baseline demineralization values. Additionally, the AgNP test group showed significant improvement over the fluoride and artificial saliva groups at 7 days and 14 days post demineralization. Demineralized and Baseline values showed no significant differences across all groups (Figure 7).

When considering the data, it is clear that the enamel samples lost approximately $95 \%$ of their initial microhardness on average. On presentation, samples became very white and chalky. Penetration via explorer was observed with moderate force, replicating severely demineralized enamel in vivo. The AgNP group showed over $100 \%$ remineralization from its starting demineralized baseline at 14 days. It was also interesting to note that in the AgNP group, microhardness improvement was rapid, showing approximately $85 \%$ of its initial gains within a 7 -day period. Figure 8 shows the relative rate of change (Remineralization Rate) of the AgNP group compared to the artificial saliva control, and Figure 9 shows the relative rate of change (Remineralization Rate) of the AgNP group compared to the fluoride group. Raw data averages for all groups are shown in Table 1. Net remineralization percentages for all groups from their demineralized baselines are shown in Table 2. Figures 5-7 show sample indentation, as well as the relative size of the indentations based on the magnification required for visualization. At its peak hardness, $1.8 \mathrm{~K}$ magnification was required to visualize the indentations. Upon demineralization, indentations only required 400X magnification. After remineralization, 600X magnification was required for the AgNP group, showing that significant micro hardness improvements had occurred.

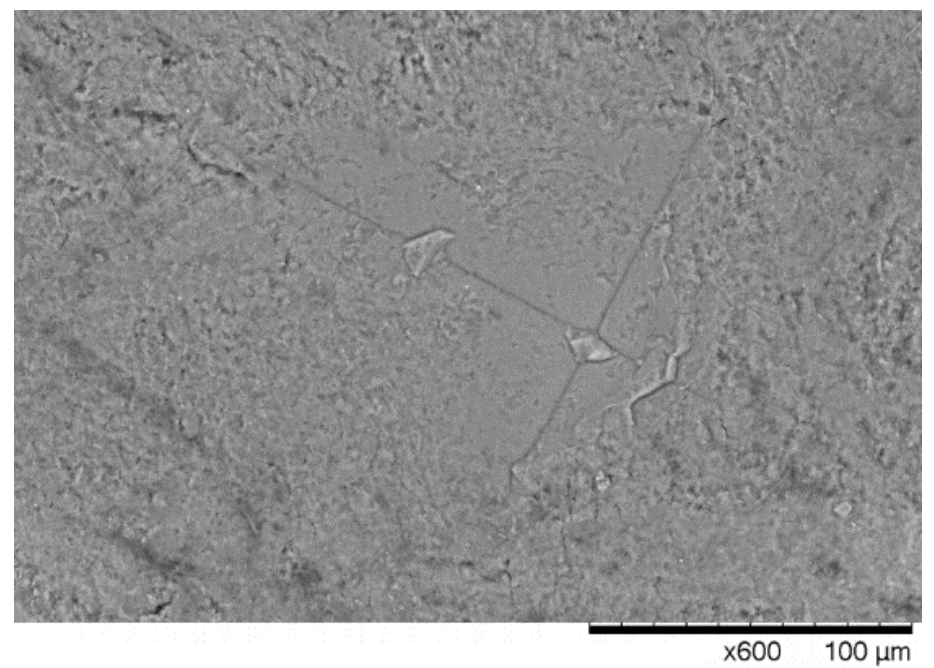

Figure 7: Sample indentation after remineralization (AgNP rinse 14 Days).

Relative Rate of Change: AgNP/Control:

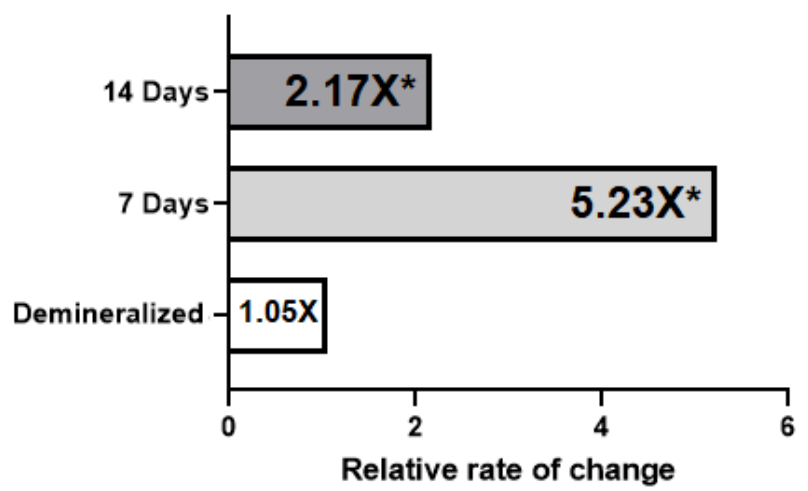

Figure 8: Remineralization rate over time (AgNP rinse/Artificial Saliva control). *Indicates significance $\mathrm{p}<0.05$.

\section{Relative Rate of Change: AgNP/Fluoride:}

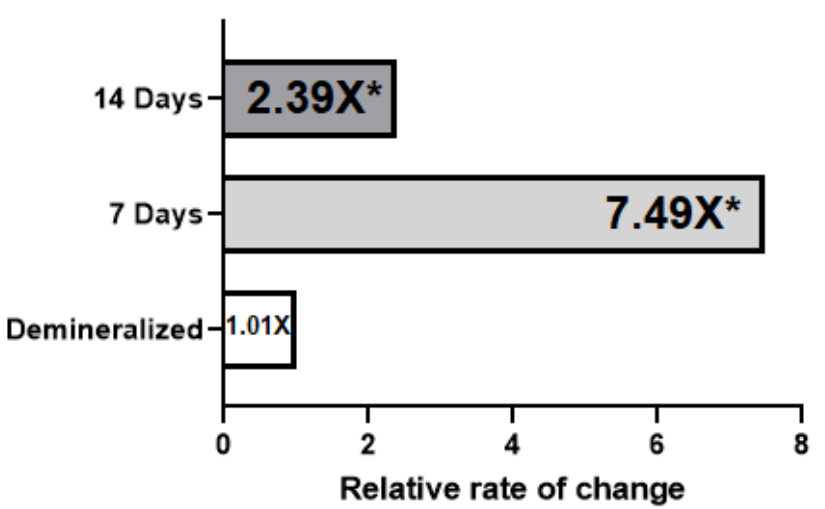

Figure 9: Remineralization rate over time (AgNP rinse/Fluoride rinse). ${ }^{*}$ Indicates significance $\mathrm{p}<0.05$.

It was interesting to note that the novel rinse containing silver nanoparticles remineralized at a much faster rate which was $2.39 \mathrm{X}$ that of the fluoride and 2.17X the negative artificial saliva control group at 14 days. In addition, the net remineralization of the novel AgNP rinse was over $100 \%$ of its post demineralized baseline, achieving a $256 \%$ net improvement over the fluoride group, and a $330 \%$ net improvement over the negative control group at the 14 day interval respectively. As can be seen from Figures 5-7, although a return of normal morphology did not occur, disorganized hole 
Table 2: Net remineralization percentage over time. *Significant change p $<0.05$ compared to demineralized values within the same group, $\nabla$ Significant change $p<0.05$ compared to other groups microhardness changes at the specified time interval.

\begin{tabular}{ccc}
\hline Groups & 7 Days & 14 Days \\
\hline Control $(n=15)$ & $13.82 \%$ & $32.92 \% *$ \\
\hline Fluoride rinse $(n=15)$ & $9.64 \%$ & $42.49 \% *$ \\
\hline AgNP rinse $(n=15)$ & $85.61 \% * \nabla$ & $108.92 \% * \nabla$ \\
\hline
\end{tabular}

like morphology became much less visible as remineralization occurred for the AgNP test group. Comparison of remineralization over time for all groups can be visualized in Figure 4. It should be noted that the relative rate of remineralization of the novel AgNP rinse reached a maximum at 7 days against the fluoride group (7.49X), and a maximum at 7 days against the control group (5.23X) respectively shown in Figures 8 and 9.

\section{DISCUSSION}

With any hygiene system it is critical to try to match the properties of saliva. Saliva is essential for providing a degree of saturation of calcium, phosphate and hydroxide ions. As a result, saliva acts as a buffer, and provides essential minerals to maintain enamel and dentinal complexes from acidic insults [19]. Much like how a restoration is designed to be as close to the properties of the original tooth structure, the aim of hygiene systems should be to match or enhance the existing properties of saliva. These functions include a neutral to alkaline $\mathrm{pH}$, buffering capacity, and the delivery of critical minerals directly to HAP. As a result, new and novel systems utilizing AgNPs could provide promise. Although the findings were significant, it is recommended that a larger study be done in order to fully evaluate the potential of this novel AgNP rinse at varying levels of AgNP concentration and demineralization values. It would also be interesting to see more detailed surface morphology to help confirm the theoretical mechanism of action stated by the authors.

\section{CONCLUSION}

In this study, it was shown that severely demineralized enamel exposed to a novel rinse containing AgNPs showed improvement over traditional remineralization systems. Although the mechanism of action for remineralization by AgNPs is not well known in vitro, the results warrant further research. As new materials become available, efforts to optimize prevention strategies should be the primary focus of dental research.

\section{ACKNOWLEDGEMENTS}

This paper is thanks to the contributions and discussions of the authors on many occasions. We are thankful for all of the authors individual contributions. We are also thankful to the University of Utah materials characterization lab for sample testing, and Nanocomposix for TEM and UV-VIS Analysis.

\section{Conflict of interest statement}

All authors acknowledge interest in Novus Research, and the surrounding patent applications for the novel nanoparticles. Funding for the study was provided by Novus Research.
1. Galdiero S, Falanga A, Vitiello M, Cantisani M, Marra V, Galdiero M. Silver nanoparticles as potential antiviral agents. Molecules. 2011;16(10):8894-8918.

2. Borzabadi-Farahani A, Borzabadi E, Lynch E. Nanoparticles in orthodontics, a review of antimicrobial and anti-caries applications. Acta Odontol Scand. 2014;72(6):413-417

3. Scarpelli BB, Punhagui MF, Hoeppner MG, Almeida RSC, Juliani $\mathrm{FA}$, Guiraldo RD, et al. In vitro evaluation of the remineralizing potential and antimicrobial activity of a cariostatic agent with silver nanoparticles. Braz Dent J. 2017;28(6):738-743

4. Aoba T. The effect of fluoride on apatite structure and growth. Crit Rev Oral Biol Med. 1997;8(2):136-153.

5. Poureslami H, Hoseinifar R, Khazaeli P, Hoseinifar R, Sharifi $H$, Poureslami P. Changes in the Concentration of Ions in Saliva and Dental Plaque after Application of CPP-ACP with and without Fluoride among 6-9 Year Old Children. J Dent Biomater. 2017;4(1):361-366

6. Dawes C. What is the critical $\mathrm{pH}$ and why does a tooth dissolve in acid? J Can Dent Assoc. 2003; 69(11):722-724.

7. The Society for Sedimentary Geology (SEPM). Clay Minerals for Petroleum Geologists and Engineers.Copyright, (SC22), 1988. Vol illustrate. SEPM; 2012.

8. Johnston CT, Schoonheydt RA. Surface and Interface Chemistry of Clay Minerals. 2006.

9. Bell LC, Posner AM, Quirk JP. The point of zero charge of hydroxyapatite and fluorapatite in aqueous solutions. J Colloid Interface Sci. 1973;42(2):250-261.

10. Di Giulio M, Di Bartolomeo S, Di Campli E, Sancilio S, Marsich E, Travan A, et al. The effect of a silver nanoparticle polysaccharide system on streptococcal and saliva-derived biofilms. Int J Mol Sci. 2013;14(7):13615-13625

11. Cheng L, Zhang K, Weir MD, Melo MA, Zhou X, Xu HH. Nanotechnology strategies for antibacterial and remineralizing composites and adhesives to tackle dental caries. Nanomedicine (Lond). 2015;10(4):627-641.

12. Callister C, Callister M, Nolan M, Nolan R. Anti-caries potential of silver nanoparticles via modulation of free calcium activity within the plaque fluid of the oral biofilm: a pilot study. Dentistry. 2018;8:12.

13. Callister C, Callister M, Nolan M, Nolan R. Cytotoxicity of novel plant-based silver nanoparticles on fibroblasts for use in dental and medical applications. Japan J Res. 2020;1(1):1-5.

14. Mäkinen KK. Sugar alcohols, caries incidence, and remineralization of caries lesions: a literature review. Int J Dent. 2010;2010:981072.

15. Featherstone J. Dental caries: a dynamic disease process. Australian Dental Journal. 2008;53(3):286-291.

16. Buzalaf MAR, Hannas AR, Kato MT. Saliva and dental erosion. J Appl Oral Sci. 2012;20(5):493-502.

17. Mei ML, Nudelman F, Marzec B, Walker JM, Lo ECM, Walls AW, et al. Formation of Fluorohydroxyapatite with Silver Diamine Fluoride. J Dent Res. 2017;96(10):1122-1128.

18. Patel J, Anthonappa RP, King NM. Evaluation of the staining potential of silver diamine fluoride: In vitro. Int J Paediatr Dent. 2018;28(5):514-522.

19. Dawes C. How much saliva is enough for avoidance of xerostomia? In: Caries Research. 2004:236-240.

\section{REFERENCES}

